\title{
Effective Pacing Intervention by Closed-loop Stimulation Using a Coronary Vein Lead in a Post-tricuspid Valve Replacement Patient
}

\author{
Yukitoshi Ikeya, Toshiko Nakai, Nobuhiro Murata, Masaki Monden, Akihito Ogaku, \\ Koichiro Hori, Ryuta Watanabe, Masaru Arai and Yasuo Okumura
}

\begin{abstract}
:
To avoid the negative effects associated with pacing, pacemakers are designed to achieve a pacing cadence as close to physiological pacing as possible. In closed-loop stimulation (CLS; a type of rate-responsive functionality used in pacemakers), the changes in impedance (which correlates with the contractility of the myocardium around the lead tip electrode) are tracked, and the paced heart rate is adjusted accordingly. We herein report a case in which we implanted a pacemaker in a post-tricuspid valve replacement patient. A ventricular lead positioned in the coronary vein exhibited good CLS functionality, and the patient's dizziness and heart failure improved.
\end{abstract}

Key words: closed-loop stimulation, CLS, coronary vein lead, left ventricular pacing

(Intern Med 59: 963-966, 2020)

(DOI: 10.2169/internalmedicine.4018-19)

\section{Introduction}

The design of pacemakers aims for pacing that is as physiological as possible in order to prevent the detrimental effects of pacing on the cardiac function and to improve the patient's exercise tolerance. Biotronik ${ }^{\circledR}$ pacemakers use a unique closed-loop stimulation (CLS) technology as a form of rate-responsive functionality.

CLS is a technology that extracts changes in impedance, which correlates with the contractility of the myocardium around the lead tip electrode, and converts the changes into a heart rate response in order to maintain the cardiac output, enabling more natural, physiological rate responsivity.

We herein report a case in which we placed the pacing lead and left ventricular (LV) lead [for cardiac resynchronization therapy (CRT)] of a pacemaker with CLS functionality in the patient's coronary vein when implanting a singlechamber ventricular (VVI) pacemaker in a post-tricuspid valve replacement (TVR) patient with chronic atrial fibrillation (AF) with bradycardia. The lead, which was positioned on the epicardial side of the LV, exhibited good CLS func- tionality.

\section{Case Report}

The patient was a 79-year-old Japanese woman who visited the emergency room at our hospital for presyncope and nausea in June 2019. As the monitor showed bradycardia associated with chronic AF and because the patient's subjective symptoms were persisting, she was admitted to the hospital.

The patient had undergone pulmonary vein isolation (PVI) for paroxysmal AF in 2010, which progressed to chronic AF. In addition, TVR was performed in March 2016 for tricuspid valve insufficiency. In other systems, endoscopic polypectomy for early gastric cancer and stent placement for lower extremity arteriosclerosis obliterans (ASO) were both performed in 2016. The patient's medical history included chronic heart failure, chronic obstructive pulmonary disease, hypertension, chronic renal failure, pulmonary hypertension, and diabetes.

The physical findings at the initial visit included the following: body temperature $36.7{ }^{\circ} \mathrm{C}$, blood pressure $182 / 80$ 

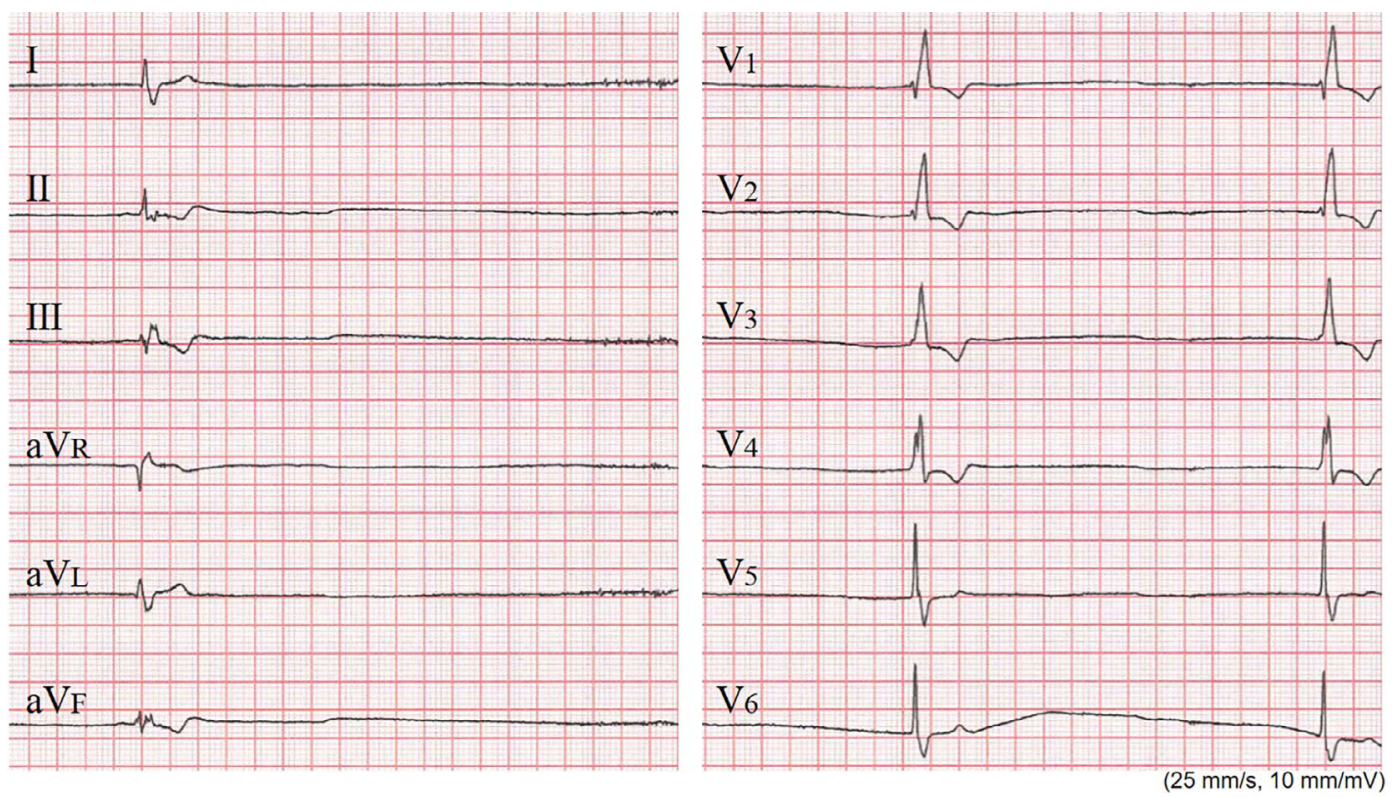

Figure 1. The electrocardiography at the initial visit. The patient's heart rate was 20 beat per minute, and complete right bundle branch block (QRS: $144 \mathrm{~ms}$ ) was observed.

$\mathrm{mmHg}$, pulse rate 45 beats per minute (bpm), irregular; and no heart murmurs or abnormal respiratory sounds. Her blood test results showed chronic anemia $(\mathrm{Hb} 9.4 \mathrm{~g} / \mathrm{dL})$ and renal dysfunction [blood urea nitrogen (BUN) $27.7 \mathrm{mg} / \mathrm{dL}$, Cr1 $15 \mathrm{mg} / \mathrm{dL}]$. Her thyroid function was within the normal range [thyroid-stimulating hormone (TSH), $2.32 \mu \mathrm{IU} / \mathrm{mL}$; free triiodothyronine (FT) $3,2.51 \mathrm{pg} / \mathrm{mL}$; free thyroxine (FT) $4,1.38 \mathrm{ng} / \mathrm{dL}]$. There were no abnormalities in cardiac enzyme levels [creatine kinase (CK), CK-muscle brain (MB), troponin I] and no changes in markers of inflammation, and the results of liver function tests were normal. NTproBNP was high at $8,464 \mathrm{pg} / \mathrm{mL}$.

Electrocardiography (ECG) revealed AF [heart rate (HR): 20-50 bpm] and complete right bundle branch block (QRS= $144 \mathrm{~ms}$ ). Inverted $\mathrm{T}$ waves were seen in leads II, III, aVF, and V1-4 (Fig. 1). Chest X-ray showed cardiomegaly (cardiothoracic ratio: $61 \%$ ) and pulmonary congestion.

Echocardiography did not exhibit clear asynergy in LV wall motion: the LV ejection fraction (LVEF) was 69\%, the LV end-diastolic dimension (LVDd) was $54 \mathrm{~mm}$, the LV end-systolic dimension (LVDs) was $33 \mathrm{~mm}$, the interventricular septal thickness at end-diastole (IVSTd) was $10 \mathrm{~mm}$, and the posterior LV wall thickness (PWTd) was $10 \mathrm{~mm}$. The findings indicating valvular disease included trivial tricuspid regurgitation (TR) (post-TVR), mild to moderate pulmonary regurgitation $(\mathrm{PR})[\mathrm{PR}$ end-pressure gradient $(\mathrm{PG})$ : $3 \mathrm{mmHg}$ ], right atrial and ventricular (RA and RV) enlargement, and left atrial (LA) enlargement with a left atrial diameter (LAD) of $47 \mathrm{~mm}$.

After admission to the hospital, a $\beta$-blocker (bisoprolol fumarate, $0.625 \mathrm{mg}$ ) that the patient had been taking was withdrawn, and she was followed-up with diuretic dose adjustments. However, as the patient frequently had a heartbeat pause lasting 5-8 seconds associated with lightheadedness and since her bradycardia did not improve, permanent pacemaker implantation was performed. Because the patient's AF had become chronic, a VVI pacemaker was used. An LV lead for CRT - instead of an RV lead - was inserted in the coronary sinus (CS) and placed on the LV side (CS lateral branch), since the patient had previously undergone TVR, although a biological tissue valve had been used.

At the implantation, the R-wave sensing was $12.1 \mathrm{mV}$, the ventricular pacing threshold was $0.6 \mathrm{~V}$ at $0.4 \mathrm{~ms}$, and the lead impedance was $526 \Omega$. All measurements were within the normal range. The pacemaker implanted this time was a model with a CLS function (Edora 8 SR-T; Biotronik, Berlin, Germany), and the settings of VVI-CLS were a lower rate of $60 \mathrm{ppm}$ and sensor rate of $120 \mathrm{ppm}$. After the pacemaker implantation, the doses of the patient's oral medications, including the $\beta$-blocker and diuretics, were adjusted once more. Her lightheadedness and heart failure signs both improved, and the patient was discharged 10 days after the implantation.

An inspection of the pacemaker and ECG Holter monitoring after the patient's pacemaker implantation indicated that even though pacing was being performed by a lead placed on the epicardial side of the coronary vein, the CLS functionality was good, as indicated by an increased pacing rate (Fig. 2) and the following data: total heart beats (/day), 100,108; maximum HR (bpm), 121; minimum HR (bpm), 59; average HR (bpm), 70; and longest RR interval (sec), 1.22 (Table). Post-pacemaker implantation chest X-ray performed after the patient's discharge did not show pulmonary congestion (Fig. 3).

\section{Discussion}

We implanted a VVI pacemaker in a post-TVR patient 

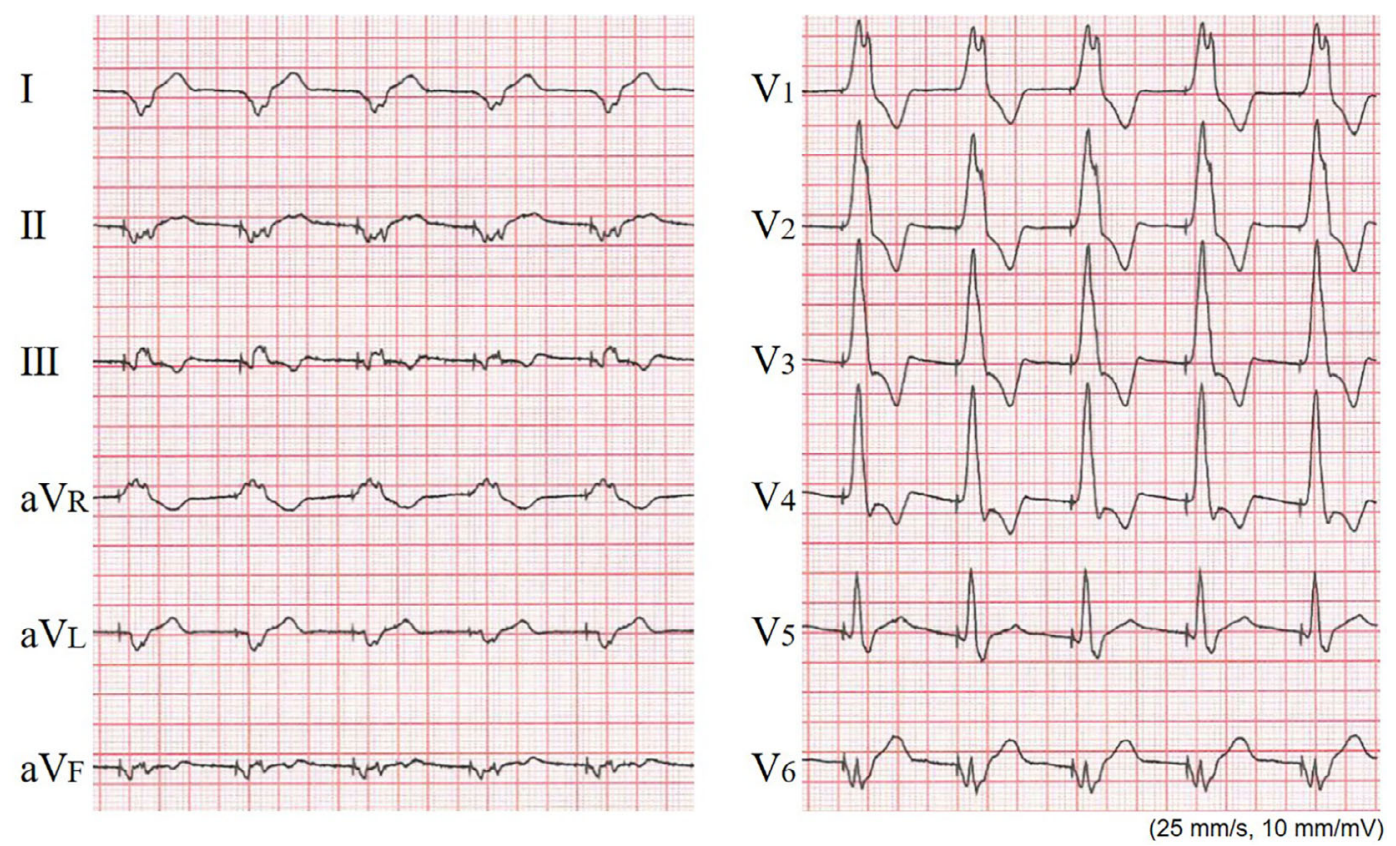

Figure 2. The post-implantation electrocardiography at rest under pacing. The pacemaker settings were VVI-closed-loop stimulation (CLS) mode, basic rate of 60, and sensor rate of $120 \mathrm{ppm}$. The heart rate is 77 beat per minute, indicating that the CLS function is working.

Table. Results of 24-hour Holter ECG Monitoring after Pacemaker Implantation.

\begin{tabular}{lc}
\hline Total heart beats (/day) & 100,108 \\
Maximum heart rate $(\mathrm{bpm})$ & 121 \\
Minimum heart rate $(\mathrm{bpm})$ & 59 \\
Average heart rate $(\mathrm{bpm})$ & 70 \\
Longest RR interval $(\mathrm{sec})$ & 1.22 \\
\hline ECG: electrocardiography, bpm: & beats per \\
minute
\end{tabular}

with AF and bradycardia. As the patient had previously undergone TVR, although a bioprosthetic valve was used, a Vlead was placed in her left ventricle using an LV lead for CRT. A pacemaker with a CLS function was selected, which worked with an LV lead.

To achieve a rate-responsive function, sensors other than the CLS were used in the present case, including an accelerometer that responds to body movements and has a superior rate response during exercise at a high activity level, and a minute ventilation sensor that monitors respiration and is suitable for responding to slow exercise and changes in mental activity.

The present patient had AF with bradycardia as her underlying heart condition; she had undergone TVR surgery and had a history of hospitalization for heart failure. The use of $\beta$-blockers for her heart failure treatment worsened the bradycardia, and the patient developed heart failure again. The pacemaker implantation stabilized the patient's heart rate, and the dose adjustments of oral drugs, including the $\beta$-blocker, alleviated her heart failure.

As noted above, in patients who have had bradycardia or develop bradycardia or chronotropic incompetence induced by a $\beta$-blocker, backup pacing at a physiological rate is important for preventing heart failure. CLS uses a sensing algorithm that can detect variations in cardiac contractility and respond to a decrease in the blood pressure by increasing the heart rate (1). Several research groups reported that CLS pacing is beneficial for patients with recurrent vasovagal syncope who demonstrate a cardio-inhibitory response on the head-up tilt table test (HUTT) (1-4). Another report showed that CLS is effective during states of emotional stress in patients with $\mathrm{AF}$ and chronotropic incompetence (5).

For these reasons, CLS was considered appropriate in this patient, who was 79 years old with an activity level that was not high and who had an unstable heartrate associated with AF and exhibited signs of heart failure. CLS is normally used with endocardial right ventricular leads, and whether or not the sensor functions well in epicardial pacing with a lead positioned in the coronary vein, as in our patient's case, was unclear. However, we selected Biotronik's Edora 8 SR$\mathrm{T}$ model because previous reports noted that CLS was capable of heart rate modulation in pediatric patients when connected to an epicardial lead $(6,7)$; in addition, we considered that, should CLS prove ineffective, the use of the rate response function of the accelerometer included in this model would be a useful contingency plan.

The CLS function was useful for LV pacing with an LV lead placed in the coronary vein of our adult patient. Our experience in this patient's case suggests the possibility of 

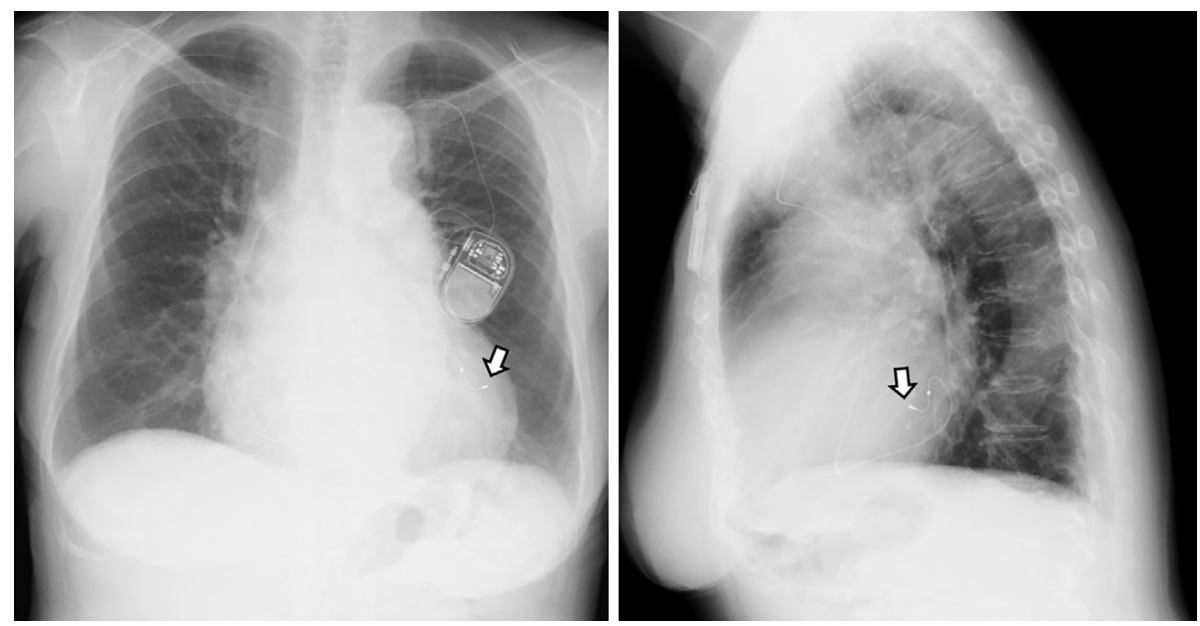

Figure 3. Post-implantation X-ray images taken at the outpatient clinic after discharge. Posteroanterior (left) and lateral (right) views. A pacing lead (arrow) placed in the coronary vein (lateral branch) is shown. No findings indicating pulmonary congestion or pleural effusion are seen.

capitalizing on the advantages of CLS in postoperative dialysis patients and patients who require a surgically placed epicardial lead.

\section{Author's disclosure of potential Conflicts of Interest (COI).}

Toshiko Nakai: Honoraria, Abbott Medical, Biotronik Japan and Medtronic Japan.

\section{References}

1. Ruzieh M, Grubb BP. Vasovagal syncope - role of closed loop stimulation pacing. Trends Cardiovasc Med 28: 534-538, 2018.

2. Ruzieh M, Ghahramani M, Nudy M, et al. The benefit of closed loop stimulation in patients with cardioinhibitory vasovagal syncope confirmed by head-up tilt table testing: a systematic review and meta-analysis. J Interv Card Electrophysiol 55: 105-113, 2019.

3. Palmisano P, Dell'Era G, Russo V, et al. Effects of closed-loop stimulation vs. DDD pacing on haemodynamic variations and occurrence of syncope induced by head-up tilt test in older patients with refractory cardioinhibitory vasovagal syncope: the tilt testinduced response in closed-loop stimulation multicentre, prospec- tive, single blind, randomized study. Europace 20: 859-866, 2018.

4. Russo V, Rago A, Papa AA, et al. The effect of dual-chamber closed-loop stimulation on syncope recurrence in healthy patients with tilt-induced vasovagal cardioinhibitory syncope: a prospective, randomised, single-blind, crossover study. Heart 99: 16091613, 2013.

5. Proietti R, Manzoni G, Di Biase L, et al. Closed loop stimulation is effective in improving heart rate and blood pressure response to mental stress: report of a single-chamber pacemaker study in patients with chronotropic incompetent atrial fibrillation. Pacing Clin Electrophysiol 35: 990-998, 2012.

6. di Pino A, Agati S, Bianca I. Efficacy of closed-loop stimulation with epicardial leads in an infant with congenital atrioventricular block. Europace 10: 334-335, 2008.

7. di Pino A, Caruso E, Censi F, Gaudenti G, Gargaro A, Calcagnini G. Physiological rate adaptation in a child with chronotropic incompetence through closed-loop stimulation using epicardial leads. Heart Rhythm Case Rep 2: 36-39, 2015.

The Internal Medicine is an Open Access journal distributed under the Creative Commons Attribution-NonCommercial-NoDerivatives 4.0 International License. To view the details of this license, please visit (https://creativecommons.org/licenses/ by-nc-nd/4.0/).

\section{(C) 2020 The Japanese Society of Internal Medicine} Intern Med 59: 963-966, 2020 